Preprint from Nucl. Instr. Meth. in Physics Research B 206 (2003) 941-946.

\title{
LATTICE LOCATION OF Fe IN DIAMOND
}

\author{
K. Bharuth-Ram ${ }^{1}$, U. Wahl ${ }^{2}$ and J.G. Correia ${ }^{3}$ \\ ${ }^{1}$ School of Physics, University of Natal, Durban 4041, South Africa \\ ${ }^{2}$ Instituto Tecnológico e Nuclear, Sacavém, Portugal \\ ${ }^{3}$ ISOLDE Collaboration, CERN, Geneva, Switzerland
}

\begin{abstract}
Incorporation of radioactive ${ }^{59} \mathrm{Fe}$ atoms in diamond was achieved by implanting precursor ${ }^{59} \mathrm{Mn}$ isotopes, with an energy of $60 \mathrm{keV}$ and to a dose of $8 \times 10^{12} \mathrm{~cm}^{-2}$, into a natural type IIa diamond sample at the online isotope separator ISOLDE at CERN. The lattice sites taken up by the $\mathrm{Fe}$ atoms, following ${ }^{59} \mathrm{Mn} \beta^{-}$-decays, have been investigated using the emission channeling (EC) technique.

Channeling measurements were made on the electrons emitted in the ${ }^{59} \mathrm{Fe} \beta^{-}$decay. Data were collected with a two-dimensional Si pad detector, along $\langle 110\rangle,\langle 100\rangle$ and $\langle 111\rangle$ axial directions from the as-implanted sample and after annealing in vacuum at $600 \mathrm{~K}, 900 \mathrm{~K}$ and $1250 \mathrm{~K}$. The channeling effects showed considerable enhancement with annealing. Fits to the observed patterns with simulations based on the many beam formalism of electron motion through a crystal lattice, show that, after annealing at $1250 \mathrm{~K}, 65(10) \%$ of the $\mathrm{Fe}$ atoms are located at sites with a projected mean displacement $\leq 0.2 \AA$ from substitutional sites.

(PACS: 61.80.-x, 61.80.-Jh, 68.55.Ln, 81.20-n)
\end{abstract}

Keywords: Fe, diamond, lattice sites, channelling.

\section{Contact author :}

K. Bharuth-Ram, School of Physics, University of Natal, Durban 4041, South Africa

Email: bharuthramk@nu.ac.za; Fax.: + 2731 2616550; Tel.: +27 312602775

\section{Introduction}

Ion beam modification studies of diamond have been prompted by the several extreme but outstanding properties of diamond, such as band gap of $5.4 \mathrm{eV}$, high thermal conductivity, high heat capacity, high electron and positive carrier mobility, which make it potentially an ideal candidate for the fabrication of semiconducting devices with a wide range of applications. Amongst the natural diamonds the type IIb, with low levels of substitutional boron impurities are $p$-type semiconducting. However, $n$-type doping has been more difficult to achieve. In the case of silicon, transition metals of the $3 d$ group introduce deep acceptor and donor levels, and are easily incorporated into the lattice by thermal diffusion near its melting point. These metals, hence, are also expected to be potential dopant candidates in diamond since its crystal is isostructural with that of silicon. The problem that requires to be addressed is that of incorporating the dopant atoms on good lattice sites where they can be electrically active. In diamond, however, its metastable nature as well as the high formation energy of potential dopants make thermal diffusion inapplicable. Ion implantation offers an attractive alternative, provided the implantation induced lattice damage can be annealed.

There exist several studies on transition metals in diamond. Electron paramagnetic resonance (epr) studies [1] show that Ni takes substitutional sites in diamond (no EPR study of $\mathrm{Fe}$ in diamond has been reported). Studies on ion implanted diamond with Moessbauer 
spectroscopy (MS) (see Ref.[2] and references contained therein) show spectra that have been generally fitted with a combination of broad doublets, indicative of considerable lattice damage in the immediate vicinity of the probe atom Speculative site assignments have been made on the basis of theoretical calculations of the isomer shifts.

No direct measurements of the lattice sites taken up by Fe in diamond, nor of the site occupancy, have been reported. Molecular orbital calculations on Fe sites in diamond by Lowther [3] found substitutional $\mathrm{Fe}\left(\mathrm{Fe}_{\mathrm{S}}\right)$ to be more energetically favoured compared with the $\mathrm{Fe}$ at a tetrahedral interstitial site $\left(\mathrm{Fe}_{\mathrm{i}}\right)$. More generally, the stability of transition metals as point defects in diamond has been examined in ab initio calculations by Johnstone et al. [4]. For $\mathrm{Fe}$, the enthalpy for $\mathrm{Fe}_{\mathrm{i}}$ and $\mathrm{Fe}_{\mathrm{S}}$ were found to be practically the same $(-20 \mathrm{eV})$, but the elastic strain energies for $\mathrm{Fe}_{i}$ were found to be fractionally higher than for $\mathrm{Fe}_{S}$.

In this contribution we report on our use of emission channeling (EC) measurements to directly determine the lattice location of ion implanted $\mathrm{Fe}$ in diamond, and on the annealing of implantation induced lattice damage. As probe we used radioactive ${ }^{59} \mathrm{Fe}$ which was incorporated in the diamond lattice by implanting precursor ${ }^{59} \mathrm{Mn}$ isotopes at the online isotope separator ISOLDE at CERN. For near-surface implantation in diamond the critical implantation dose is found to be $10^{14}-10^{15} \mathrm{~cm}^{-2}$, beyond which the lattice graphitizes [5]. Hence, in our study the implantation dose was kept below $10^{13} \mathrm{~cm}^{-2}$.

\section{Experimental Details}

In electron emission channeling measurements [6 - 8] radioactive isotopes are implanted into single crystal samples, and the yield of beta particles or conversion electrons emitted in the decay of probe atoms is measured outside the sample as a function of angle relative to the principal axial directions. Channeling effects on the emitted electrons by the positively charged atomic rows of the crystal lattice lead to anisotropic emission yields whose angular pattern is characteristic of the lattice site occupied by the emitter. Simulations based on the many beam formalism of electron diffraction through the crystal are then fitted to the experimental channeling patterns to determine the identity of the sites and the fraction of probe atoms located at a particular site.

As Fe probes we used ${ }^{59} \mathrm{Fe}$, which were produced by implanting parent isotopes ${ }^{59} \mathrm{Mn}$ with an energy of $60 \mathrm{keV}$ into single crystal type IIa diamonds, at the online isotope separator ISOLDE at CERN. ${ }^{59} \mathrm{Mn}$ decays by $\beta^{-}$emission $\left(\mathrm{Q}_{\beta}=5190 \mathrm{keV}, \mathrm{t}_{1 / 2}=4.6 \mathrm{~s}\right)$ to the ground state of ${ }^{59} \mathrm{Fe}$ which in turn $\beta^{-}$decays, with $\mathrm{t}_{1 / 2}=44.5$ days, primarily to the 1291.6 and 1099.3 keV states of ${ }^{59} \mathrm{Co}$.

The ${ }^{59} \mathrm{Mn}$ ions were implanted through a $1 \mathrm{~mm}$ diam. aperture into a type IIa diamond sample polished with its face parallel to the (110) plane. Channeling data were collected several days after the implantation (i.e. after the short-lived ${ }^{59} \mathrm{Mn}$ precursors had decayed to ${ }^{59} \mathrm{Fe}$ ), from the as-implanted sample (room temperature) and after annealing under vacuum for 10 mins. at temperatures of $600 \mathrm{~K}, 900 \mathrm{~K}$ and $1250 \mathrm{~K}$. The $\beta^{-}$-particles emitted in the ${ }^{59} \mathrm{Fe}$ decay were detected in a position sensitive Si detector of $30 \times 30 \mathrm{~mm}^{2}$ area, as described in Ref. [8].

\section{Analysis and Discussion}

Channeling patterns from ${ }^{59} \mathrm{Fe}$ were extracted for $\beta^{-}$particles in the energy window $50-500$ $\mathrm{keV}$, which includes practically all the $\beta^{-}$particles, but avoids $\mathrm{x}$-rays, emitted by the sample. The contribution of backscattered electrons to the data for each of axial directions investigated were estimated by Monte Carlo simulations and subtracted from the events recorded by the Si pads. Figure 1 shows the channeling yields from the single crystal diamond after annealing at $1250 \mathrm{~K}$. Prominent channeling effects along the $\langle 111\rangle$, $\langle 100\rangle$ and $\langle 110\rangle$ axial directions 
and $\{111\}$ planar direction are clearly evident, indicating that the emitters are located close to substitutional sites.

In order to identify the lattice location more precisely the experimental channeling patterns were fitted with combinations of theoretical yields for various lattice sites. The theoretical simulations were carried out within the many beam formalism, based on the dynamical theory of electron diffraction through a crystal lattice $[6,7]$. Quantum mechanical diffraction effects result in a rich fine structure in the angular dependence of the emission yields, hence necessitating simulations in a fine mesh of small angular steps. We therefore considered $x$ - and $y$-angular ranges of $+/-3.0^{\circ}$ around the $\langle 110\rangle,\langle 100\rangle$ and $\langle 111\rangle$ directions, in steps of $\Delta x, \Delta y=0.05^{\circ}$, and obtained theoretical patterns of electron emission probability, $\mathrm{X}_{\text {theor }}(\vartheta, \varphi)$, where $\vartheta$ and $\varphi$ denote polar and azimuthal angles from the axis. Patterns were calculated in energy steps of $25 \mathrm{keV}$ over the electron energy window of 50$500 \mathrm{keV}$, and summed according to the total $\beta^{-}$- yield, and smoothed using a Gaussian of $\sigma=$ $0.1^{\mathrm{o}}$ to account for contribution to the angular resolution of the $1 \mathrm{~mm}$ beam spot on the sample and the size and shape of the detector pad.

The theoretical emission patterns were fitted to the experimental yields $\mathrm{X}_{\exp }$ according to

$\mathrm{X}_{\text {exp }}(\vartheta, \varphi)=S\left\lfloor f_{1}\left(\mathrm{X}_{\text {theor }, 1}(\vartheta, \varphi)-1\right)+f_{2}\left(\mathrm{X}_{\text {theor }, 2}(\vartheta, \varphi)-1\right)+1\right\rfloor$

where $S$ is a scaling factor common to all angles in a pattern, and $f_{\mathrm{i}}$ denote the fraction of emitter atoms at sites. The remaining fraction, $f_{\mathrm{R}}=1-\left(f_{1}+f_{2}\right)$, the so-called 'random fraction, accounts for emitters that produce isotropic emission yields and which may be at sites of low symmetry or of extensive damage.

The sites modeled included substitutional $(\mathrm{S})$, tetrahedral interstitial $(\mathrm{T})$, hexagonal interstitial $(\mathrm{H})$, bond center $(\mathrm{BC})$, anti-bonding $(\mathrm{AB})$, split $\langle 100\rangle$, and displacements along $\langle 111\rangle$ and $\langle 100\rangle$ directions between these sites, and rms displacements of 0.05 to $1.0 \AA$ from $\mathrm{S}$ and $\mathrm{T}$ sites. An ideal S site did not give a satisfactory fit to the data. Best fit results (shown in Fig.1) were obtained for a fraction $66(5) \%$ of the Fe atoms located at sites displaced a distance of $0.25(2) \AA$ from the substitutional site along $<111>$ directions, and the rest on sites that give isotropic emission yields. Both $\langle 111\rangle$ displacements from $\mathrm{S}$ towards $\mathrm{BC}$ and $\mathrm{AB}$ sites gave equally good fits. Good fits were also obtained for $65 \%$ of the emitters at an $\mathrm{S}$ site but with an isotropic rms displacement of $u=0.17(3) \AA$. It is evident that for small displacements from regular lattice sites the channeling effects cannot give unique identification of the displacement, but give information only on the mean projected displacement perpendicular to the channeling direction.

Fig. 2(a) shows the channeling effect observed in the $\langle 110\rangle$ axial direction, as a function of annealing temperature, while the near-S site fractions determined from fits to the data are displayed in Fig. 2(b). The electron emission channeling effects which were already evident directly after room temperature implantation, showed enhancement after annealing at $600 \mathrm{~K}$, and could be well fitted by assuming 30-35\% of the Fe on near-S sites, with the remainder contributing an isotropic emission yield. Self interstitials in diamond become mobile above $600 \mathrm{~K}$, and the partial recovery of the diamond lattice observed in our measurements may be attributed to this effect. Annealing at $1250 \mathrm{~K}$ results in considerably further enhancement and quite prominent channeling effects along the major axial directions and the $\{111\}$ planes become clearly evident. These results are in general agreement with the annealing characteristics of implantation damage in diamond reported by Bharuth-Ram et al.[9] and Quintel et al.[10], which show two distinct annealing stages, the first setting in at $600 \mathrm{~K}$ and the second at $1200 \mathrm{~K}$.

Our results show two significant results. Firstly, that annealing results in significant healing of the radiation damage of the diamond lattice, and secondly, that after annealing at $1250 \mathrm{~K}$, about $65 \%$ of the $\mathrm{Fe}$ atoms are at near-substitutional sites. However even after the 
annealing the $\mathrm{Fe}$ atom was still remained at its position, approx. $0.20 \AA$ away from a substitutional site, and showed no evidence of any significant move towards the $\mathrm{S}$ site. EC studies on implantation sites of As, In, Cd, Ce and Gd [9-13] also show appreciable fractions of these ions to be located near substitutional sites in the lattice. However, perturbed angular correlation studies show that considerable lattice damage remains even after annealing at temperatures as high as $1600 \mathrm{~K}[11,12]$ Also casting a damper on the hopes of Fe being a potential dopant in diamond is the result of the theoretiacl calculations of Johnstone et al. [4] that the most stable site for the $3 d$ transition metals in diamond is the di-vacancy, and that $\mathrm{Fe}$ is not stable at this site.

\section{Conclusions}

Emission channeling measurements have been performed on a diamond single crystal implanted with radioactive ${ }^{59} \mathrm{Fe}$ probes. With the implantation dose kept low $\left(8 \times 10^{12} \mathrm{~cm}^{-2}\right)$ the channeling effects of the $\beta^{-}$particles emitted in the ${ }^{59} \mathrm{Fe}$ decay were already evident in the as-implanted sample. Annealing results in considerable enhancement of the electron channeling. After room temperature implantation and annealing at $1250 \mathrm{~K}, 65(5) \%$ of the $\mathrm{Fe}$ atoms occupy sites displaced from substitutional sites by up to $0.25(5) \AA$ along $<111>$ directions, or with a mean projected displacement from $\mathrm{S}$ sites of $d \approx 0.17$ (3) $\AA$. However, even after annealing at $1250 \mathrm{~K}$ the probe atoms displayed no significant tendency to move closer to the substitutional sites.

\section{Acknowledgements:}

The authors thank Bart Devries for the Monte Carlo calculations of the backscattered electron intensities. KB-R thanks the National Research Foundation (South Africa) for support, and UW acknowledges his fellowship supported by the FCT, Portugal. This work was also funded by the FCT project CERN/FIS/43725/2001 and by the European Union LSC contract HPRICT-1999-00018.

\section{References}

1. J. Isoya, H. Kanda, J.R. Norris, J. Tang and M.K. Bowman, Phys. Rev. B 41 (1990) 3905

2. K. Bharuth-Ram, M. Hartick, E. Kankeleit, C. Dorn, P. Held, R. Sielemann, L. Wende, J. Kübler and J.P.F. Sellschop, B 58 (1998)

3. J.E. Lowther, Phys. Letters 104A (1984) 273

4. K. Johnstone and A. Mainwwod, Physica B 308-310 (2001) 565

5. S. Prawer, C. Uzay-Saguy, G. Braunstein, R. Kalish, Appl. Phys. Lett. 63 (1993) 2502

6. H. Hofsäss and G. Lindner, Phys. Rep. 20 (1991) 123

7. H. Hofsäss, Hyperfine Interact. 87/98 (1996) 247.

8. U. Wahl, J.G. Correia, S. Cardoso, J.G. Marques, A. Vantomme, G. Langouche, Nucl. Instr. Meth. B136-138, (1998) 744

9. K. Bharuth-Ram, H. Quintel, M. Restle, C. Ronning, H. Hofsäss, S.G. Jahn, J. Appl. Phys. 78 (1995) 5180

10. H. Quintel, K. Bharuth-Ram, H. Hofsäss, M. Restle, C. Ronning, Nucl. Instr. Meth. B118 (1996) 72

11. J.G. Correia, J.G. Marques, E. Alves, D. Forkel-Wirth, S.G. Jahn. M. Restle, M. Dalmer, H. Hofsäss, and K. Bharuth-Ram, Nucl. Instr. Meth. 127/128 (1997) 723

12. K. Bharuth-Ram, A. Burchard, M. Deicher, H. Hofsäss, H. Quintel, M. Restle, and C. Ronning, Phys. Rev. B 64 (2001) 195207-1

13. K. Bharuth-Ram, U. Vetter, H. Hofsäss, C. Ronning and M. Dietrich, Nucl. Instr. Meth. B 190 (2002) 835 


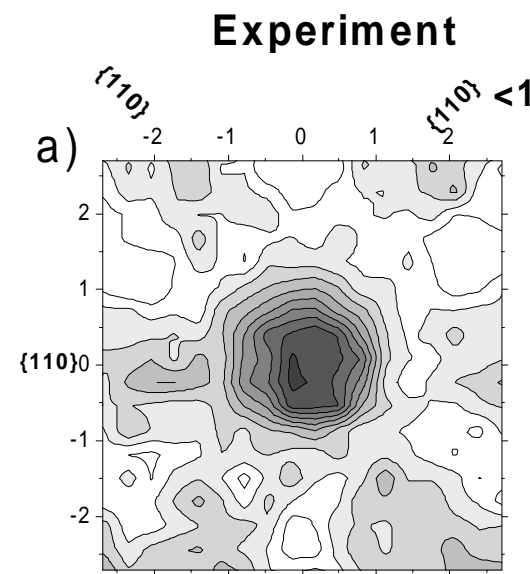

\section{Simulation}

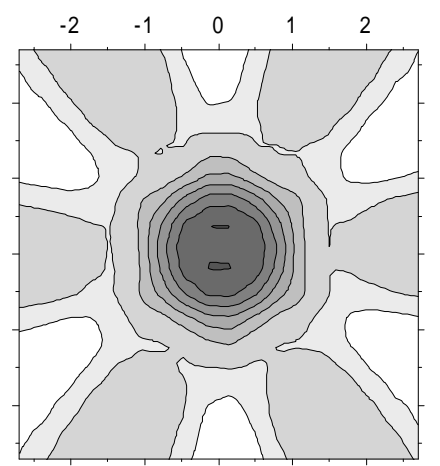

d)

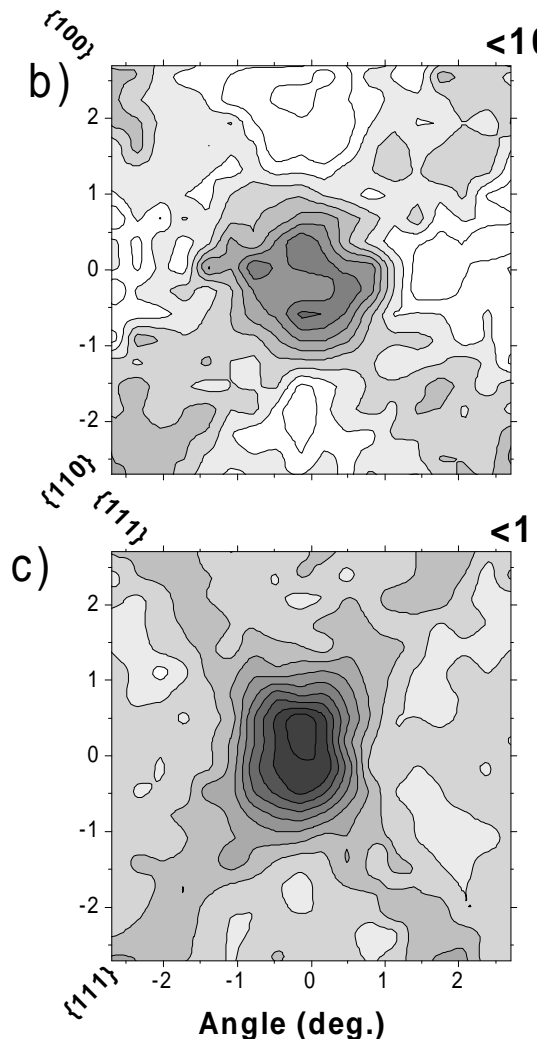

$<100>$

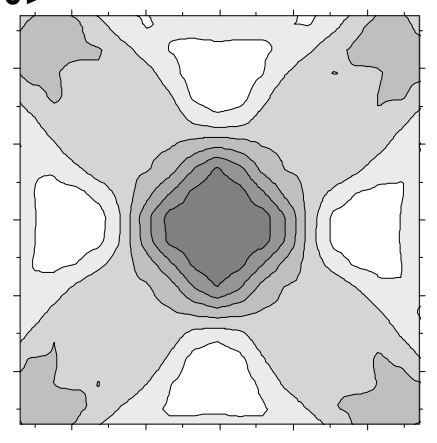

e)
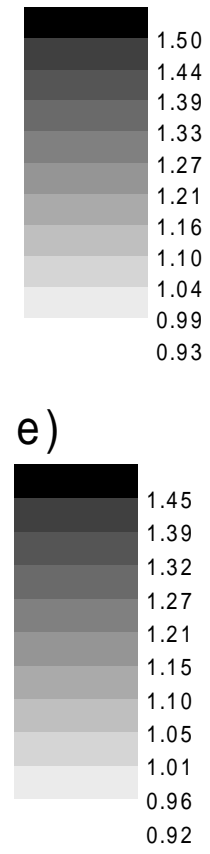

f)
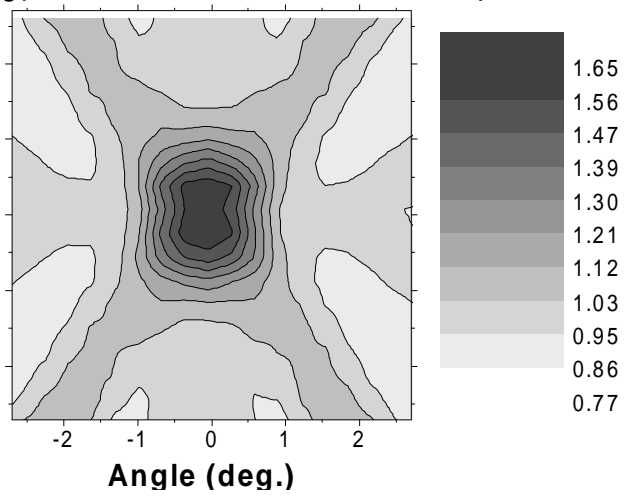

Fig. 1. Two dimensional channelling effects for $\beta^{-}$particles from ${ }^{59} \mathrm{Fe}$, observed in the $\left.<111\right\rangle$ $\langle 100\rangle$ and $\langle 110\rangle$ axial directions. Best fits to the data shown in the figure correspond to a fraction 65(5)\% of Fe atoms displaced 0.25(2) $\AA$ along <111> directions from an S site, and the remainder on random sites. 

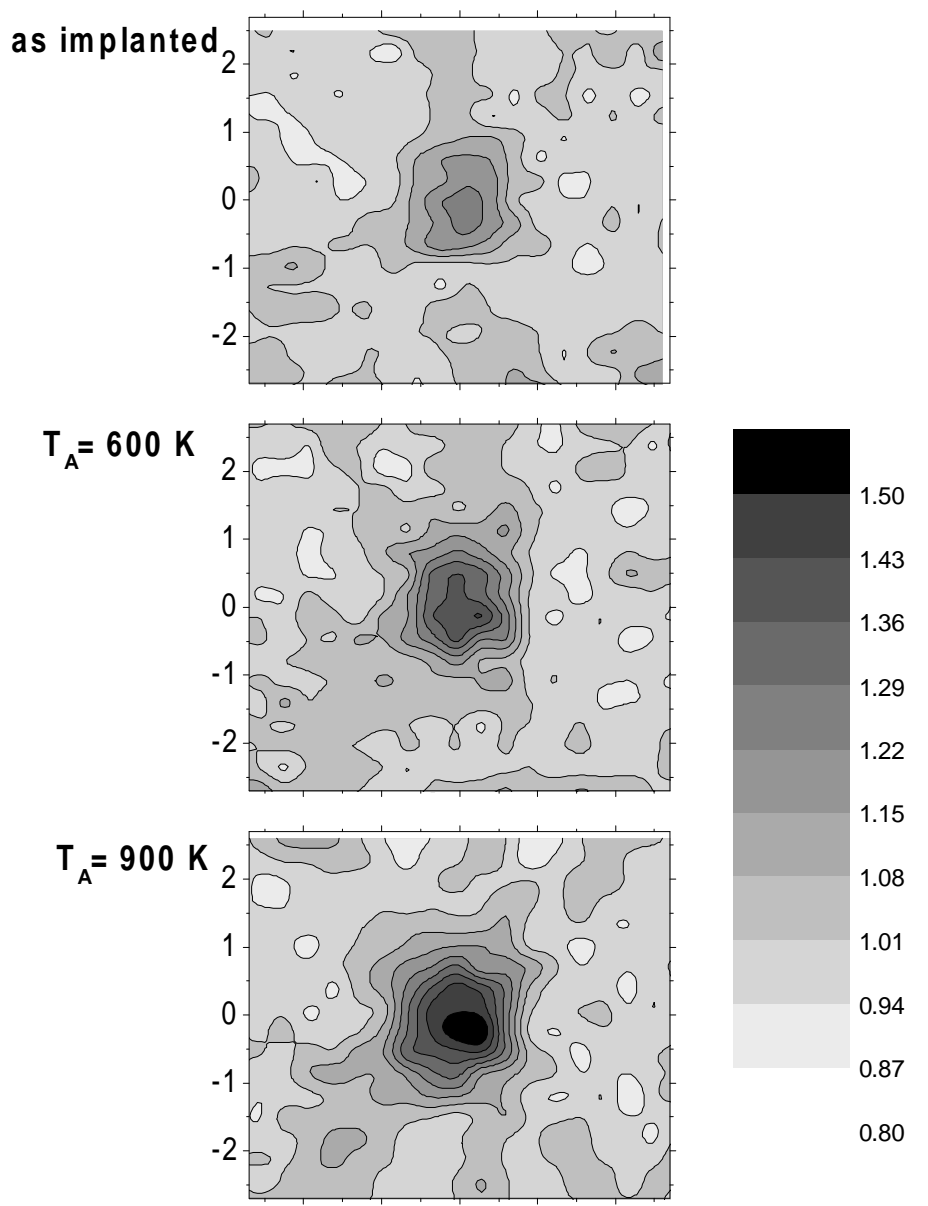

$T_{A}=1250 \mathrm{~K}$

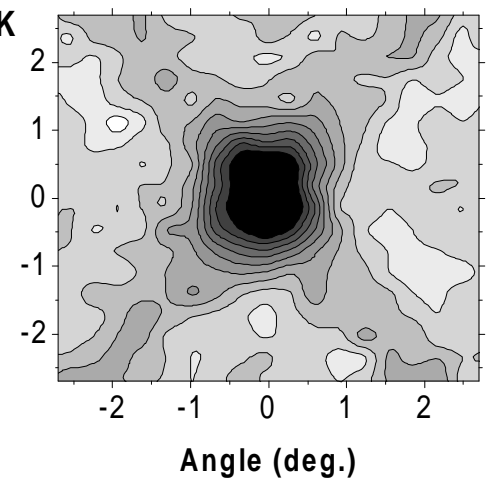

Fig. 2(a). Two dimensional channeling effects for $\beta^{-}$particles from ${ }^{59} \mathrm{Fe}$ in the $\langle 110\rangle$ axial direction, observed from the as-implanted sample and after annealing at $600 \mathrm{~K}, 900 \mathrm{~K}$ and $1250 \mathrm{~K}$. 


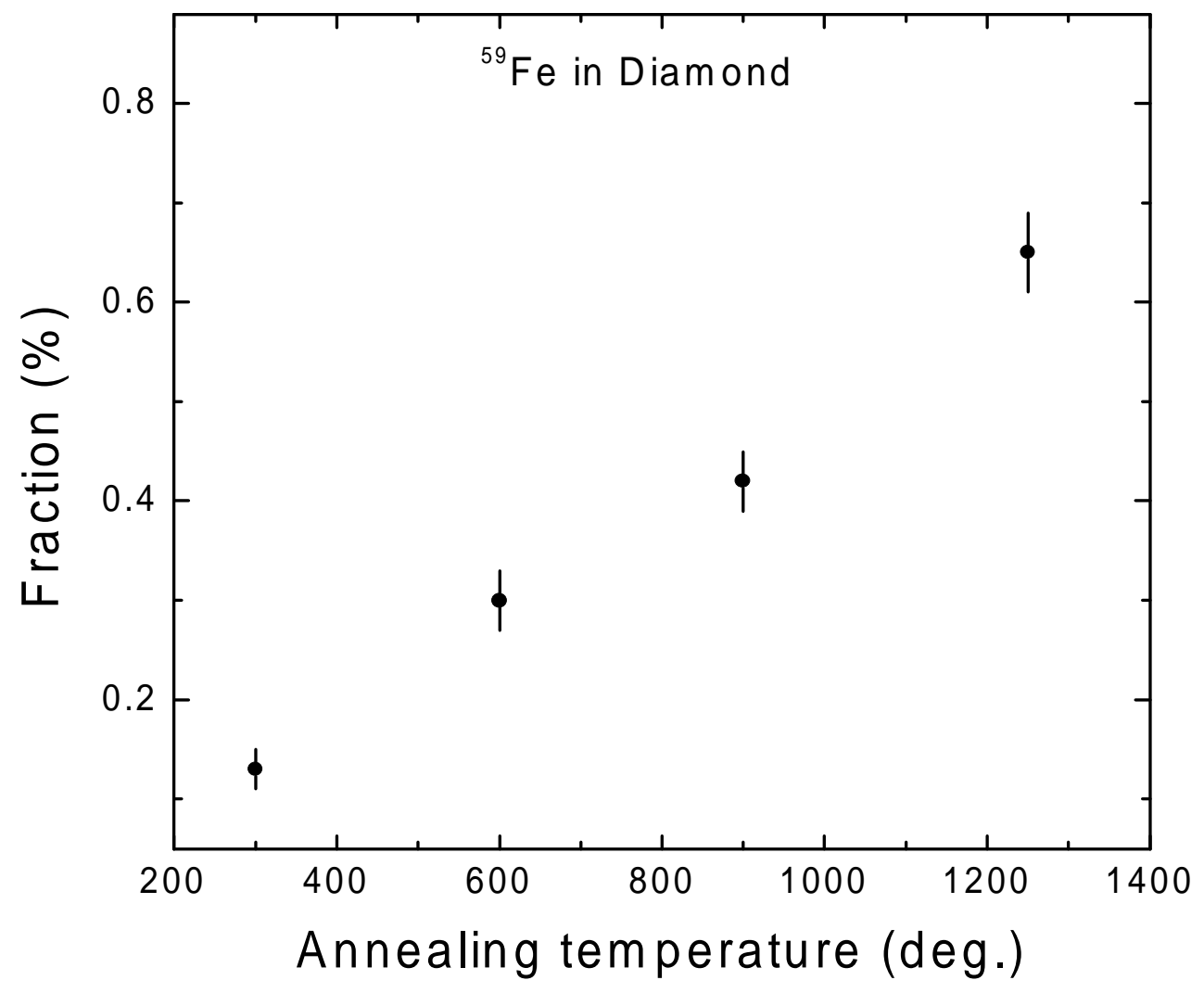

Fig. (2b) Fraction of ${ }^{59} \mathrm{Fe}$ atoms located at near-S sites as a function of annealing temperature. 